\title{
INSTALACIÓN DE ALMACENAMIENTO DE RESIDUOS RADIACTIVOS DE EL CABRIL, EN HORNACHUELOS, CÓRDOBA-ESPAÑA
}

\author{
(RADIOACTIVE WASTE DISPOSAL FACILITY OF EL CABRIL \\ IN HORNACHUELOS, CÓRDOBA-SPAIN)
}

Pablo Zuloaga Lalana, Ingeniero Industrial

Jefe del Proyecto del C.A. El Cabril (Departamento de Ingeniería)

Fecha de recepción: 24 - VI - 94

ENRESA

\section{RESUMEN}

La Instalación de El Cabril tiene capacidad para almacenar a largo plazo los residuos de baja y media actividad generados en España hasta el año 2010.

La Instalación consta de:

- Tres módulos de almacenamiento temporal, anteriormente existentes.

- Una zona de almacenamiento, con 28 celdas de hormigón armado en las que se depositan los residuos, introducidos previamente en contenedores de bormigón.

- Un edificio destinado al acondicionamiento de los residuos procedentes de las instalaciones radiactivas, de los producidos en el Centro y de los residuos compactables, asi como al reacondicionamiento de los bultos provenientes de los grandes productores.

- Unos laboratorios de verificación, destinados a la realización de ensayos destructivos y no destructivos de bultos reales y de muestras radiactivas y simuladas.

- Instalaciones complementarias destinadas a almacenamiento transitorio, vigilancia radiológica, seguridad, oficinas, talleres, servicio médico, vestuarios, lavandería, protección contra incendios, ventilación, tratamiento de agua y de aguas residuales, alimentación eléctrica, etc.

\section{SUMMARY}

The Installation of $\mathrm{El} \mathrm{Cabril} \mathrm{has} \mathrm{the} \mathrm{capacity} \mathrm{for} \mathrm{long-term}$ disposal of low and medium radioactivity waste generated in Spain by 2010.

The Installation consists of:

- Three already existing modules for temporary storage.

- A disposal area with 28 reinforced concrete cells where waste is deposited after being introduced in concrete containers.

- A building for Waste Conditioning of the waste coming from radioactive installations, waste produced in the Center, as well as the compactable waste; and for re-conditioning of the substances coming from great producers.

- Verification laboratories for destruction and non-destruction tests of real substances and simulated radioactive specimens.

- Complementary installations for temporary storage, radiological surveillance, security, offices, worksops, medical service, changing rooms, laundry, fire protection system, airconditioning system, water and sewage treatment, electrical power supply, etc. 


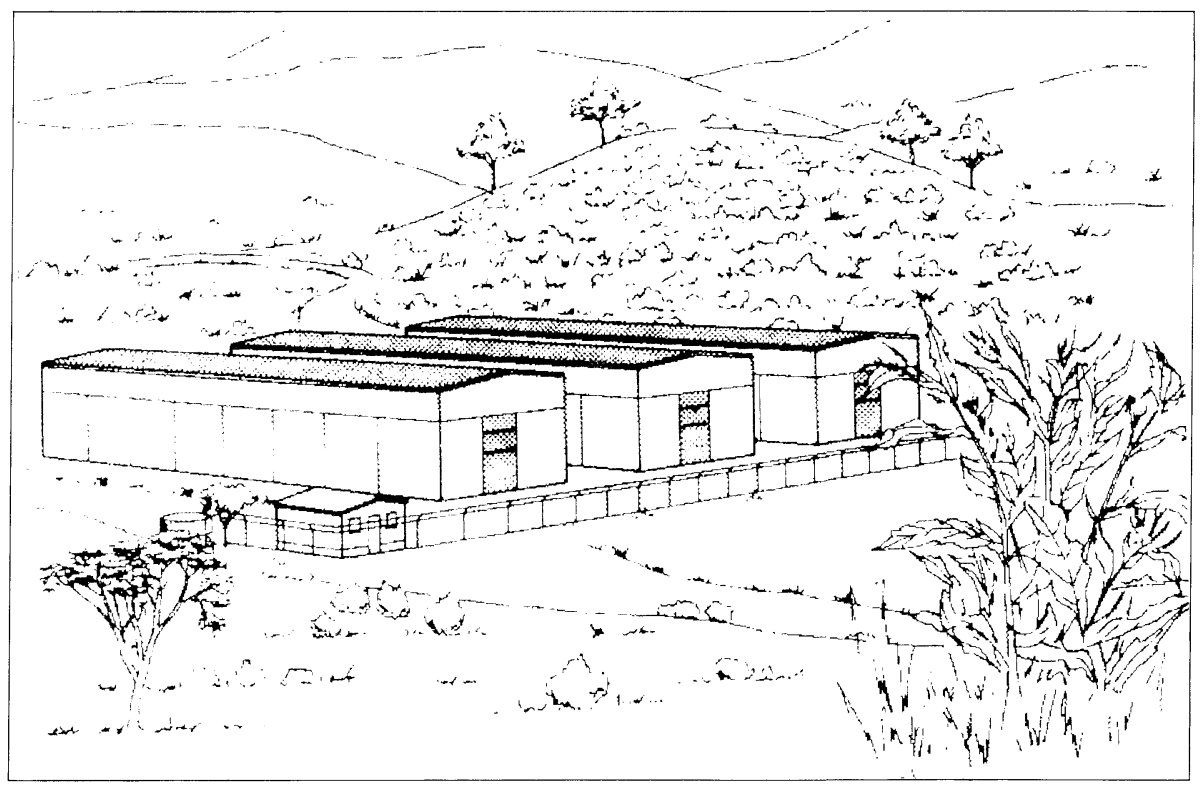

Fig. 1. Módulos de almacenamiento

\section{Introducción}

Por Orden Ministerial de 20 de octubre de 1994 (Referencia 1) se otorgó a ENRESA el Permiso de Explotación Provisional del Proyecto de Ampliación de la Instalación de almacenamiento de Residuos Radiactivos Sólidos de sierra Albarrana "El Cabril", cuya construcción se inició en enero de 1990, tras la obtención de la autorización de construcción (Referencia 2), y de la licencia municipal.

\section{Criterios generales de diseño}

Las nuevas instalaciones de El Cabril están proyectadas de modo que puedan constituir un almacenamiento a largo plazo de residuos de baja y media actividad. Para ello, han de cumplir dos objetivos fundamentales:

\section{Asegurar la protección inmediata y diferida de} las personas y del medio ambiente.

La protección inmediata no plantea problemas específicos y es similar a la de otras instalaciones. La protección diferida se asegura mediante un sistema de barreras múltiples.

2. Permitir la libre utilización del emplazamiento tras un periodo de una duración máxima de trescientos años, sin limitaciones de origen radiológico.

Esta duración máxima de trescientos años está tomada de la reglamentación francesa adoptada como referencia y corresponde a un período para el que se puede asegurar razonablemente que puede impedirse la realización de otras construcciones sobre el almacenamiento, y tras el cual se supone que todas las obras de ingeniería están totalmente degradadas.

En el Proyecto de El Cabril se ha adoptado, además, como objetivo básico la posible recuperación de los residuos, si las circunstancias lo aconsejasen. Aun cuando esto siempre es posible en un almacenamiento superficial, el proyecto original fue modificado sustancialmente para introducir este concepto.

La capacidad de almacenamiento, con la solución adoptada, es de $35.000 \mathrm{~m}^{3}$ de bidones de $0,22 \mathrm{~m}^{3}$, lo que corresponde a una capacidad de recepción de unos $42.000 \mathrm{~m}^{3}$, antes de la reducción de volumen prevista de una parte de los residuos.

De los objetivos citados anteriormente se deducen dos necesidades:

El aislamiento de los bultos de residuos frente al agua y la limitación de actividad.

El agua constituye, junto con el hombre, el posible vehículo de dispersión de la radiactividad almacenada. Por ello, se requiere aislar el almacenamiento de las aguas subterráneas (situando los bultos de residuos por encima de la capa freática) y de las aguas superficiales (evitando las zonas de inunda- 
ción y vaguadas y protegiéndolos de las precipitaciones).

Por otra parte, al adoptarse hipótesis pesimistas de intrusión humana al finalizar el período de vigilancia, con una duración máxima de trescientos años (por ejemplo, la vivienda permanente o la construcción de una gran obra pública sobre el propio almacenamiento), la actividad total y la actividad másica máxima deben limitarse, de modo que el impacto en dichas hipótesis sea aceptable. Conviene hacer hincapié en que son los escenarios de intrusión, y no los de impacto debido a posibles arrastres por el agua que pudiera infiltrarse hasta los residuos, aun suponiendo la degradación total de las barreras de ingeniería, los que limitan la actividad que puede ser almacenada en un sistema de almacenamiento superficial o a baja profundidad. Estos escenarios de intrusión son prácticamente independientes del emplazamiento elegido.

Esta limitación es la que claramente separa los residuos de baja y media actividad y vida corta, almacenables en superficie, de otras categorías cuya evacuación debe hacerse en profundidad.
Adicionalmente, el Centro tiene capacidad para el tratamiento de los residuos de pequeños productores, la reducción de volumen de los residuos compactables, del acondicionamiento de los residuos generados en la propia instalación y el reacondicionamiento de los bultos de residuos en contenedores de hormigón, previamente a su almacenamiento.

Otros criterios de diseño generales son el de vertido nulo de líquidos radiactivos y la limitación de descargas de efluentes gaseosos, de modo que la dosis en el límite del área restringida sea inferior a 0,01 $\mathrm{mSv} / \mathrm{año}$

Para el diseño de blindajes se ha adoptado un límite de $5 \mathrm{mSv} /$ año para los trabajadores. La ventilación se ha diseñado de modo que las concentraciones en aire sean inferiores al 0,1 por ciento del LCDA.

\section{El sistema de almacenamiento (Fig. 2)}

Los bultos de residuos, en su mayor parte bidones de $0,22 \mathrm{~m}^{3}$, se almacenan, como se ha indicado, en el interior de contenedores de almacenamiento, de

CENTRO DE ALMACENAMIENTO "EL CABRIL"

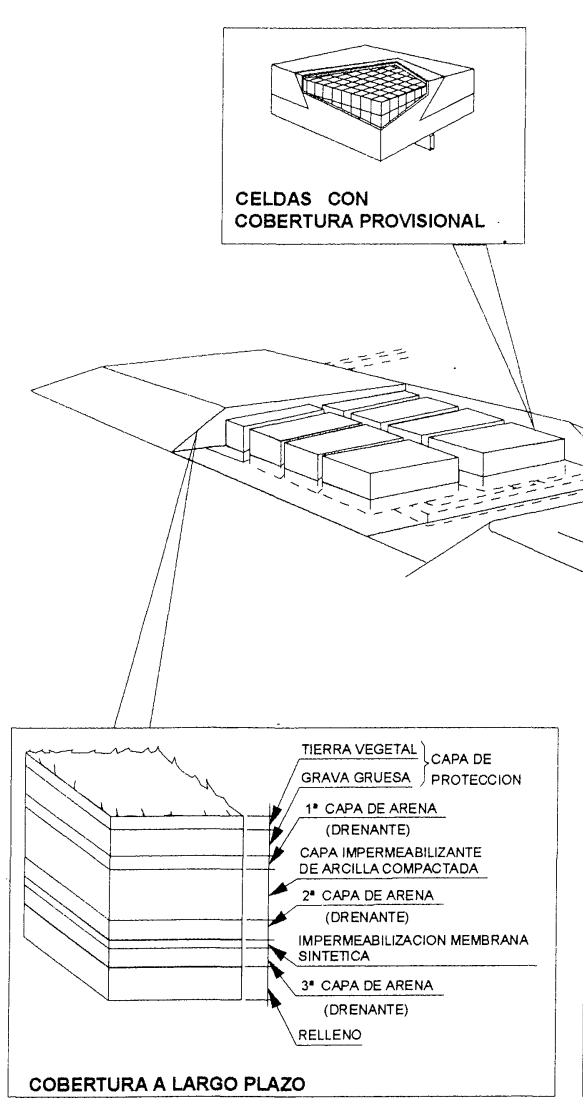

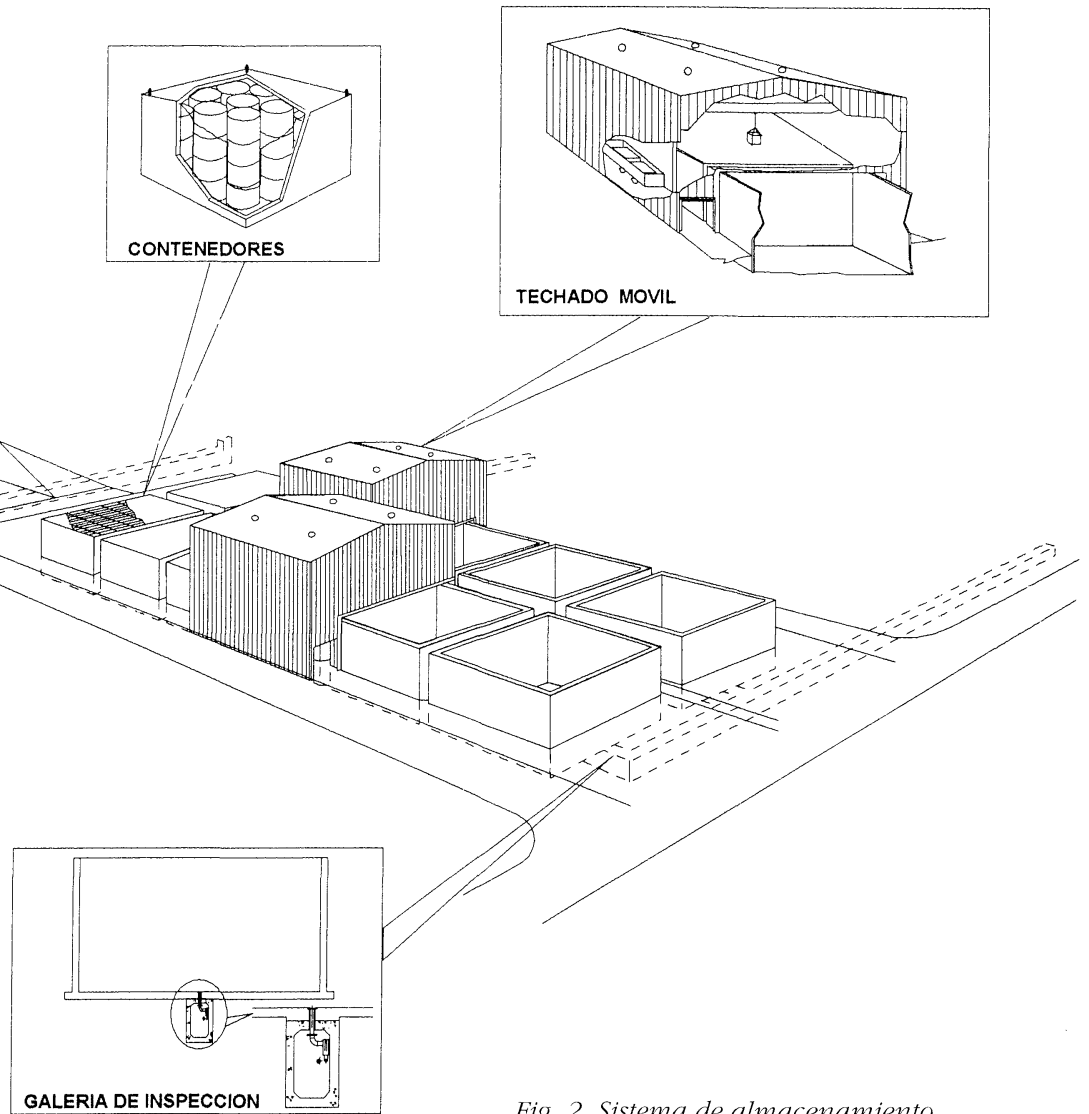

Fig. 2. Sistema de almacenamiento 


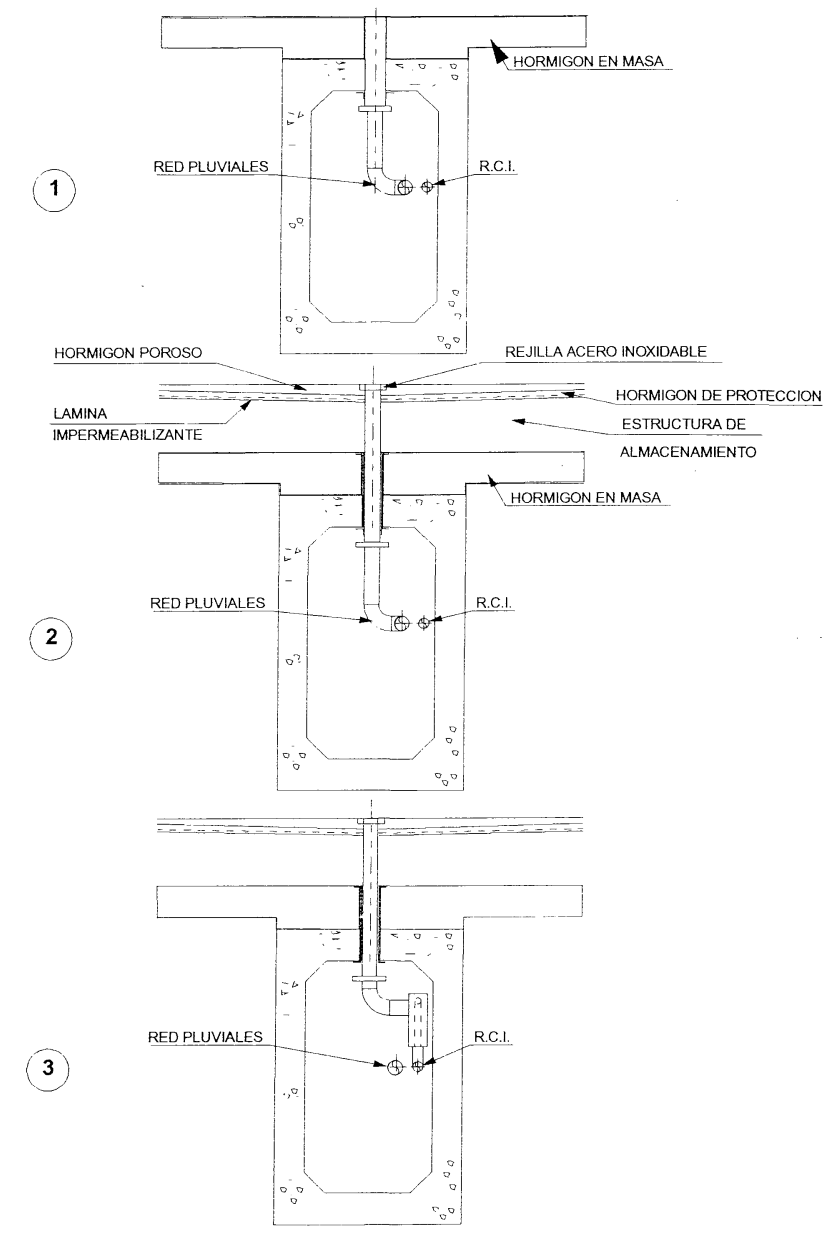

Fig. 3. Detalle plataforma. Estructura de almacenamiento

hormigón. Los bidones son inmovilizados dentro del contenedor, constituyendo un bloque de hormigón de unas $24 \mathrm{t}$ de peso, y 2,25 x 2,25 x 2,20 m de dimensiones exteriores.

Estos contenedores se almacenan apilándose en unas estructuras o celdas de almacenamiento. Cada una de éstas tiene capacidad para 320 contenedores del tipo indicado y unas dimensiones exteriores aproximadas de $24 \times 19 \times 10 \mathrm{~m}$. Los contenedores se colocan en contacto entre sí, excepto en una cruz o franja central destinada a absorber las tolerancias de fabricación o de colocación de los contenedores.

Tanto los contenedores como las celdas de almacenamiento están diseñados para soportar solicitaciones extremas, incluyendo un sismo de aceleración en el suelo de 0,24 g. La definición del hormigón utilizado en las celdas y contenedores ha sido objeto de un programa de investigación desarrollado por el Instituto Eduardo Torroja del CSIC, cuyo objetivo era la optimización de la durabilidad de las barreras de hormigón. El hormigón utilizado es de alta resistencia característica $\left(350 \mathrm{~kg} / \mathrm{cm}^{2}\right)$ y compacidad, y resistente a sulfatos y agua de mar (a pesar de las bajas concentraciones de sulfatos y cloruros en el agua del emplazamiento). La resistencia característica del hormigón de los contenedores ha sido aumentada a $413 \mathrm{~kg} / \mathrm{cm}^{2}$. Además, los contenedores han sido diseñados y probados para cumplir los requisitos de bulto tipo A e industrial tipo 3 del Reglamento de Transporte de Mercancías Peligrosas por Carretera.

Una vez completada la operación de cada celda de almacenamiento se rellena la franja central con grava, para rigidizar el conjunto y rellenar huecos, y se construye una losa superior de cierre. La estructura es entonces impermeabilizada con una capa sintética.

La placa inferior constituye el elemento principal de la celda de almacenamiento. Tiene un espesor de 0,6 $\mathrm{m}$ en los bordes y 0,5 en el centro, formando pendiente, y está cubierta de una capa impermeable de poliuretano y de una capa de 10-20 cm de hormigón poroso, para disponer de una superficie horizontal para colocación de los contenedores (Fig. 3). La placa tiene la función de recoger cualquier agua que pudiera haber infiltrado, dirigiéndola a una red de tuberías instalada en unas galerías de inspección situadas bajo las estructuras de almacenamiento. Cada estructura se une a esta red, denominada red de control de infiltraciones (RCI), a través de un depósito de retención, de modo que se permita, en caso de recogerse agua en la red de control, determinar de qué celda proviene para poder así proceder a reparar la cobertura de protección, así como tomar muestras del agua recogida (Fig. 3).

Esta red de tuberías (Fig. 4), de funcionamiento pasivo, desemboca en un depósito final de control, con capacidad de recogida de un año, teniendo en cuenta la infiltración nominal de la cobertura, más las correspondientes a hundimiento de la cobertura. De este modo se permite vigilar adecuadamente el funcionamiento del sistema de almacenamiento detectando y determinando el origen de cantidades anormales de agua infiltrada, así como una eventual contaminación de la misma.

Durante la fase de explotación y con el triple objetivo de proteger los contenedores con residuos de la intemperie, minimizar la cantidad de agua a recoger en la red de control de infiltraciones y ser- 


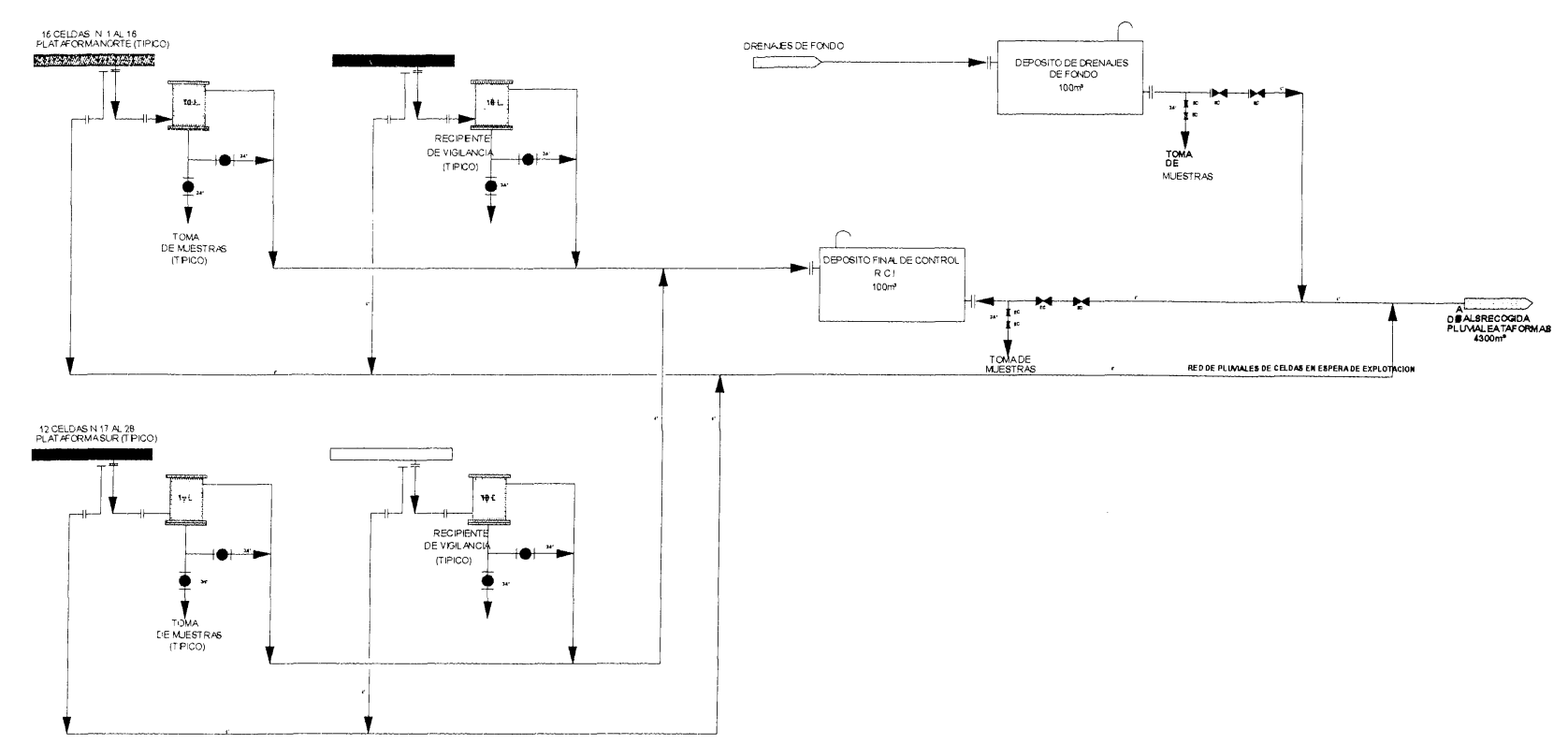

lig. 4. Red de control de infiltraciones

vir de soporte de los medios de manejo de contenedores, cada fila de estructuras de almacenamiento está servida por un techado móvil montado sobre carriles (Fig. 5). Este techado auxiliar se sitúa sobre la estructura de almacenamiento en explotación. Después de la impermeabilización de ésta, el techado se traslada a la estructura adyacente.

Una vez finalizada la explotación de El Cabril se procederá a cubrir el conjunto con una cobertura de baja permeabilidad, constituida por capas alternas de materiales impermeables y drenantes que protegerá a largo plazo los contenedores que contienen los bultos de residuos y asegurará una buena durabilidad de los propios contenedores.

Se define así el sistema multibarreras, constituido por tres barreras:

La primera está constituida por la matriz de inmovilización de los residuos que permite que, aun en caso de que los bultos se sumergieran en agua, la tasa de liberación de radionucleidos fuera mínima y el contenedor de almacenamiento que los aisla.

La segunda, formada por las estructuras de almacenamiento, la cobertura y la red de control de infiltraciones, limita el acceso de agua a los bultos y permite el control de las aguas que pudieran haber estado en contacto con los mismos y su eventual tratamiento en caso necesario.
La tercera, la barrera geológica, está constituida por el terreno del entorno y limitaría el impacto de una eventual liberación en caso accidental o en las hipótesis de degradación total de las dos primeras barreras, adoptada para la fase de libre utilización.

\section{El emplazamiento}

El Cabril está situado en el noroeste de la provincia de Córdoba, a unos $130 \mathrm{~km}$ por carretera de la capital, en un área de escasa población. Pertenece al término municipal de Hornachuelos. El acceso principal parte de la carretera de Fuente Ovejuna a Cazalla de la Sierra, al norte de la Instalación (Fig. 5).

El emplazamiento se localiza en el sur de la zona centroibérica del macizo ibérico, en una zona de sismicidad moderada.

Las nuevas instalaciones ocupan una superficie de 20 hectáreas en la parte norte de El Cabril, junto a la pista principal de acceso existente. Se sitúan en una formación geológica de gneisses y micaesquistos, denominada formación Cabril, que presenta una potencia superior a los $300 \mathrm{~m}$.

A partir de 1986, y continuando estudios iniciados por el CIEMAT se han acometido importantes investigaciones que han permitido un conocimiento detallado de este emplazamiento. Como cifras significativas hay que destacar el centenar de sondeos realizados, o los $8.000 \mathrm{~m}$ perforados en la caracterización del emplazamiento. 


\section{COMUNIDADES AUTÓNOMAS}

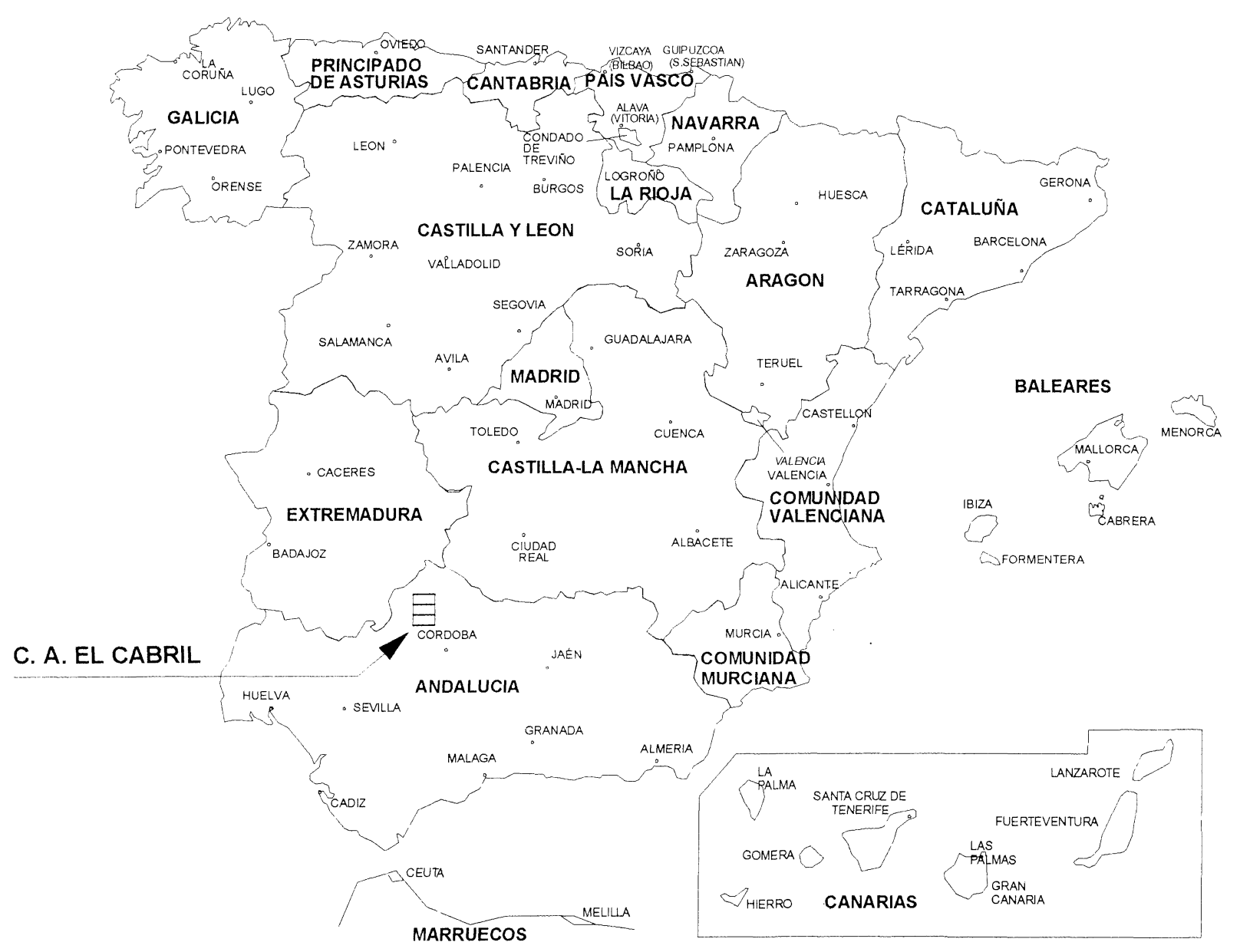

Fig. 5. Localización

Los principales estudios de emplazamiento realizados, así como los consultores principales, se indican en la Tabla 1.

Tabla 1. Estudios de emplazamiento y consultores

\begin{tabular}{|l|l|}
\hline Geología regional & INTECSA-D'APPOLONIA \\
\hline Sismicidad, riesgo sísmico & INTECSA-D'APPOLONIA \\
\hline Geología del emplazamiento & CGS \\
\hline Geotecnia & INTECSA \\
\hline Radiometría & INYPSA \\
\hline Meteorología & CIEMAT, UITESA \\
\hline Hidrología e Hidrogeología & CGS, ANDRA, BRGM, FRASA \\
\hline Microsismicidad & GEONICA, A. Bernal \\
\hline Geoquímica & CIEMAT \\
\hline Geomorfología y erosión & INYPSA \\
\hline Modelización hidrogeológica & ETSICCP Barcelona \\
\hline Búsqueda de materiales & INGEMISA \\
\hline
\end{tabular}

La zona destinada al almacenamiento de los residuos se sitúa en la cresta de un cerro y está rodea- da por dos arroyos, uno afluente del otro, formando una península y facilitando la vigilancia radiológica del emplazamiento (Fig. 6). Las celdas de almacenamiento se sitúan por encima del nivel freático y del nivel máximo de inundación previsible.

\section{Descripción general de la instalación}

Para adaptarse a la topografía existente, la instalación se ha dividido en dos zonas: la zona de almacenamiento y la zona de acondicionamiento y de edificios auxiliares. Como se ha indicado en el apartado anterior, la zona de almacenamiento ocupa la zona más adecuada para el almacenamiento a largo plazo de los residuos; los edificios auxiliares se sitúan en la ladera del macizo principal de la sierra Albarrana, en una zona separada de la anterior por un arroyo.

En la Fig. 7 se da una disposición general de la instalación. 


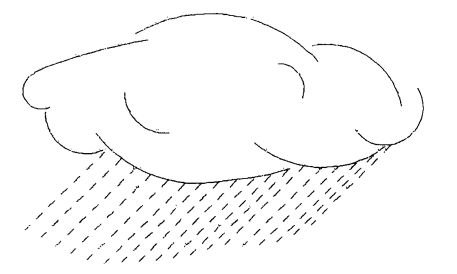

\author{
ESQUEMA DEL \\ FUNCIONAMIENTO \\ HÍDRICO DEL CERRO \\ DE LOS MORALES
}
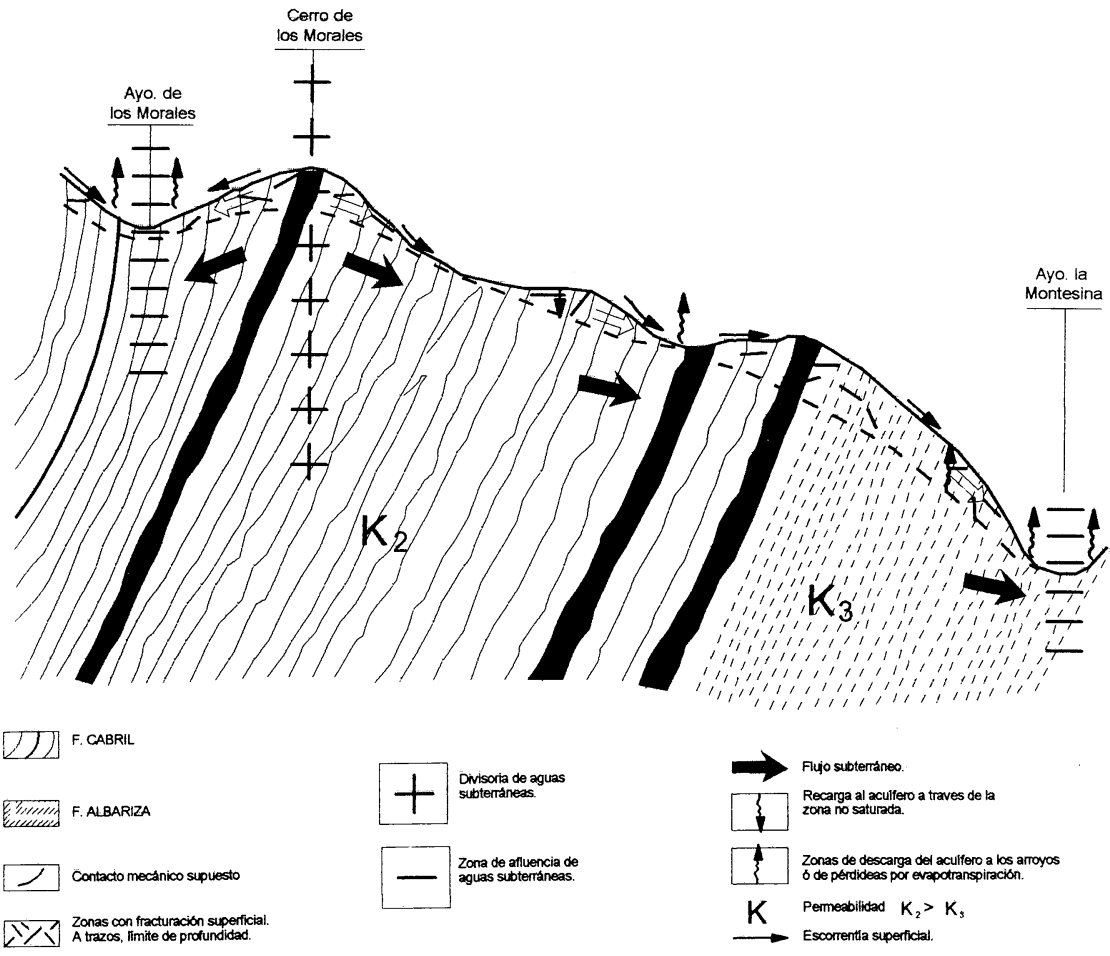

Fig. 6. Corte esquemático geológico

\subsection{Zona de almacenamiento}

En la zona de almacenamiento (Fig. 7) se han construido 28 celdas, agrupadas en dos áreas o plataformas: la plataforma norte, con 16 estructuras, y la sur, con 12. Estas plataformas constituyen superficies horizontales de unos $90 \mathrm{~m}$ de anchura, excavadas con trinchera en la loma del cerro, dejando unos taludes laterales contra los que apoyará en su momento la cobertura definitiva.

En cada una de estas áreas, las celdas están semienterradas respecto al nivel de operación y se disponen en dos filas, cada un de las cuales está servida por un techado móvil, que puede trasladarse a lo largo de los carriles instalados.

Estos techados soportan un puente grúa de $32 \mathrm{t}$ para el manejo de los contenedores. El mando de los puentes-grúa se realiza mediante control remoto desde la Sala de Control, situada en el Edificio de Acondicionamiento, permitiéndose de esta manera minimizar las dosis en la operación.
Los camiones que transportan los contenedores de almacenamiento se sitúan en el pasillo lateral existente entre las estructuras de almacenamiento y la pared del techado. Por el exterior de éste hay un vial adicional. Esta anchura, quizá innecesaria a primera vista, se requiere para permitir cubrir el almacenamiento con la cobertura impermeable, con taludes de pendiente adecuada.

En esta zona se ubican, además, el depósito de control de la red de control de infiltraciones y la balsa de recogida de pluviales.

En un área aneja, separada por la valla que delimita la zona restringida, se sitúan las plantas de preparación de hormigón y de fabricación de los contenedores de almacenamiento, así como el banco y grúa-pórtico auxiliar para realizar ensayos del TPC de bultos de hasta 30 toneladas.

Entre las diferentes opciones posibles ENRESA adoptó la de construir y operar la fábrica de contenedores en El Cabril, sobre todo para poder contro- 


\section{A. EL CABRIL (IMPLANTACIÓN GENERAL)}

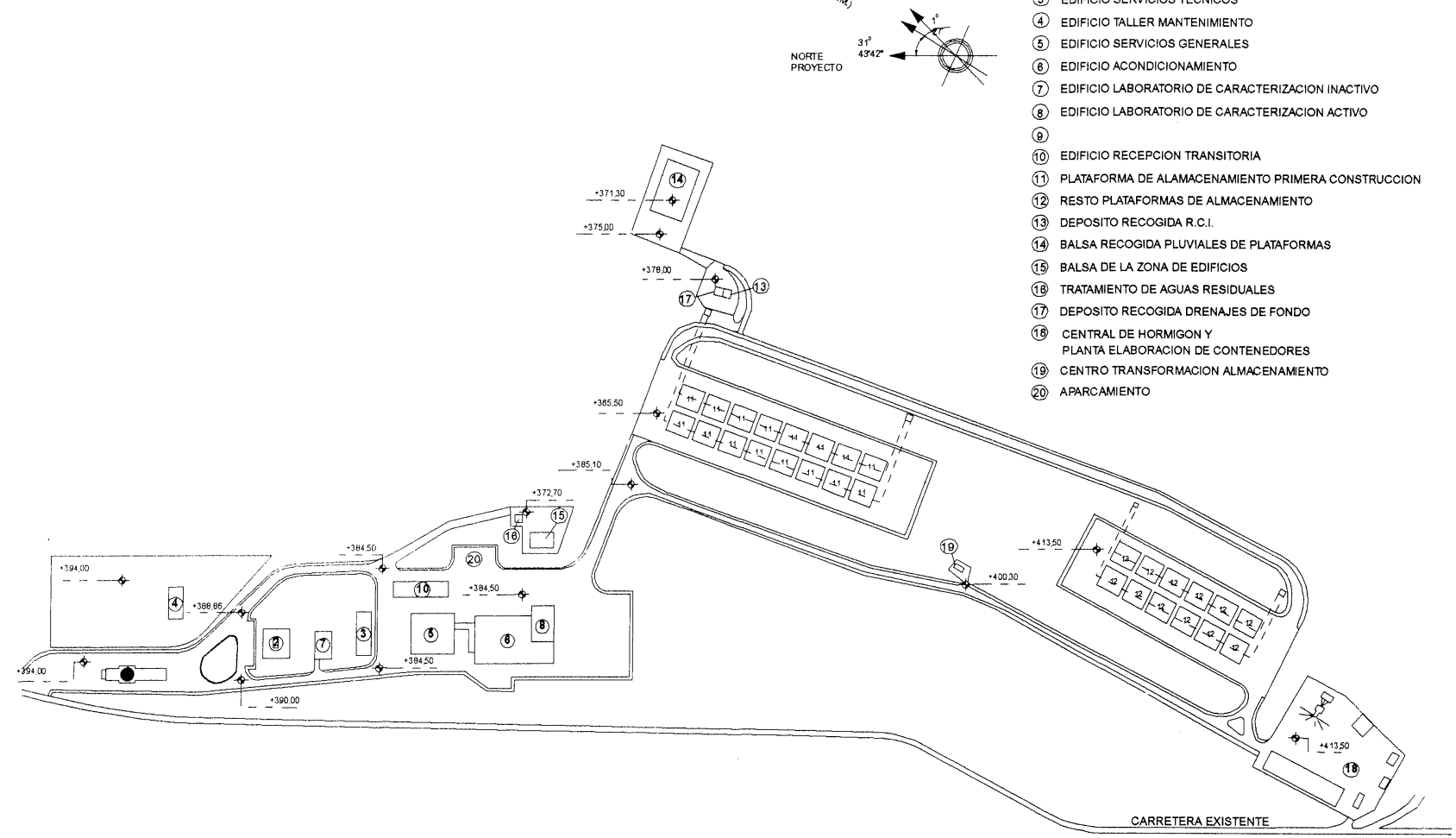

Fig. 7

lar adecuadamente la calidad de los componentes del hormigón, elemento fundamental para la durabilidad de las barreras, así como la fabricación en sí misma.

La planta de hormigones tiene capacidad suficiente para el hormigonado continuo de las losas de cierre. La planta de fabricación de contenedores dispone de dos líneas de producción, cada una de ellas con mesas de elaboración de armaduras, dos moldes metálicos abatibles de alta precisión, zona de curado húmedo y zona de curado seco. Cada línea dispone de un puente-grúa de 15 toneladas.

\subsection{Zona de edificios}

En la zona de edificios se sitúan las instalaciones auxiliares para el tratamiento y acondicionamiento de los residuos y su control, así como los servicios auxiliares necesarios para la operación y mantenimiento del Centro.

A continuación, se indican, ordenadas por su posición respecto a la entrada a la instalación, los edificios e instalaciones auxiliares (el número entre paréntesis indica la identificación dada en la Fig. 7).

\section{Edificio de seguridad industrial ${ }^{1}$}

En este edificio se agrupan el control de accesos, el puesto central de vigilancia y los medios de lucha contra incendios.

\section{Taller de mantenimiento ${ }^{4}$}

Incluye los talleres mecánico, eléctrico y de vehículos.

\section{Edificio de administración ${ }^{2}$}

En este edificio se sitúa la Dirección, las Jefaturas de los diferentes servicios y los servicios administrativos.

\section{Laboratorio de verificación. Edificio inactivo-}

Este edificio está destinado a la realización de ensayos y pruebas sobre probetas no activas de características similares a las de los diferentes tipos de bulto cuya retirada se solicita. Consiste básicamente en un laboratorio de hormigones y morteros, con cámara climática, célula triaxial, prensas, laboratorio químico, etc. 
Igualmente alberga las oficinas de personal de explotación del laboratorio de caracterización.

\section{Edificio de servicios técnicos ${ }^{3}$}

En este edificio se instalan los equipos principales de los diferentes sistemas de servicios auxiliares: centro de transformación, distribución eléctrica, diesel de apoyo, plantas de producción de frío y calor, y planta de tratamiento de agua. También alberga al panel de control de servicios auxiliares.

\section{Edificio de servicios generales 5}

En el mismo se ubican los servicios de protección radiológica, servicio médico normal y nivel I, vestuarios, lavandería, laboratorio de vigilancia ambiental, equipo de calibración de monitores de radiación, laboratorio radioquímico, equipo de contaje y control radiológico de accesos del personal. A través de este edificio se realiza el acceso a la zona vigilada de todo el personal, exceptuando a los conductores de vehículos.

\section{Edificio de recepción transitoria}

Este edificio contiene el puesto de control radiológico de vehículos y el puesto de descontaminación de vehículos, así como un almacén transitorio de bidones. Este edificio, junto con el de servicios generales, marca la separación entre la zona no reglamentada desde el punto de vista de protección radiológica y la zona vigilada.

\section{Edificio de acondicionamiento ${ }^{6}$}

En este edificio (Fig. 8) se realizan todas las operaciones de tratamiento y acondicionamiento que se describen en el apartado siguiente. Por el mismo pasan la práctica totalidad de los residuos. Alberga, además, la Sala de Control desde la que se opera la mayor parte de los sistemas de este edificio así como los equipos de manutención de la zona de almacenamiento y en la que se centraliza la información de la explotación de toda la instalación. En el Edificio de Acondicionamiento se sitúa además la distribución eléctrica de este edificio y el adyacente de Verificación Activo y los sistemas de alimentación ininterrumpida, de ventilación controlada y de vigilancia de la radiación.

Se trata de un edificio en tres plantas, si bien el sótano y la planta alta son sólo parciales. En el sótano se ubican los depósitos de recogida de efluentes líquidos y la sala de almacenamiento de residuos radiactivos inflamables. En la planta alta se sitúan los equipos de ventilación, separados en dos salas: una de equipos de impulsión (climatizadores), y otra para los filtros y ventiladores de extracción. En el apartado siguiente se describen los diferentes procesos que se realizan en este edificio.

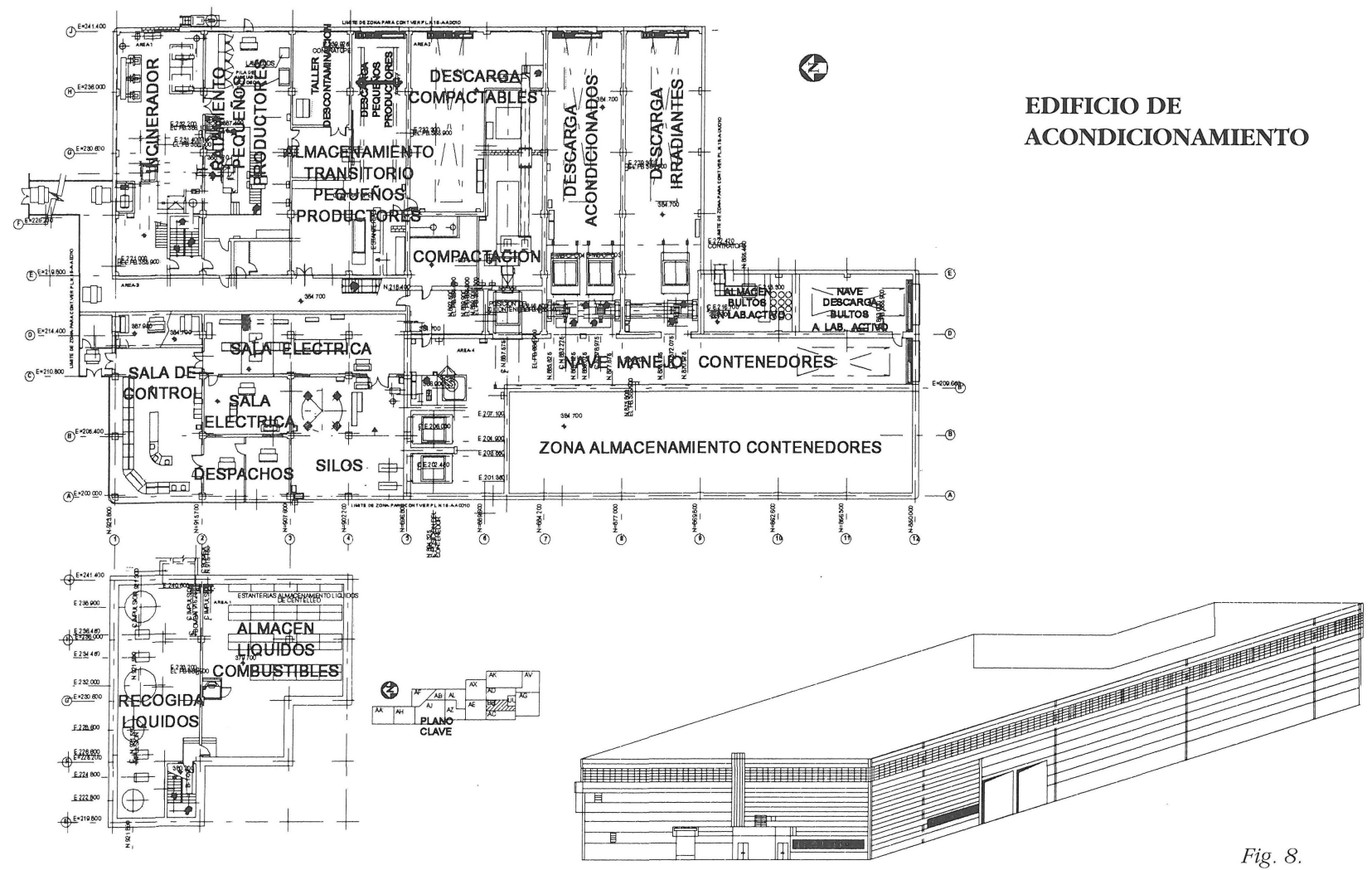

(c) Consejo Superior de Investigaciones Científicas 


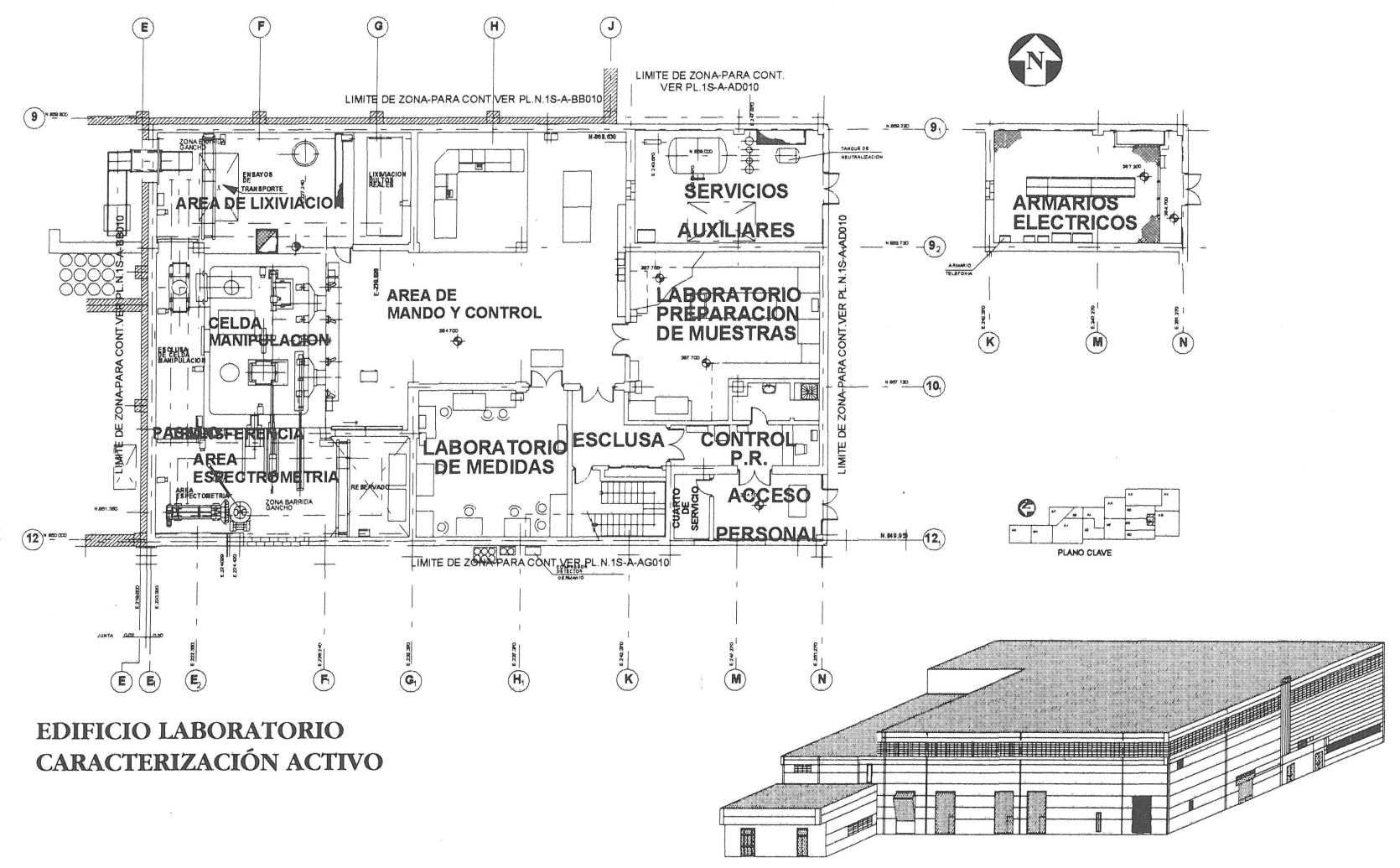

Fig. 9

Laboratorio de verificación de la calidad. Edificio activo $^{8}$

Este edificio está destinado a la realización de ensayos para determinar las características de los diferentes tipos de bultos, sobre probetas activas y bultos reales, y a la verificación técnica de algunos de los bultos que lleguen al Centro.

Igualmente se prevé realizar en el mismo trabajos de investigación y desarrollo para la optimización del proceso de solidificación de residuos.

Este laboratorio está dispuesto alrededor de una celda de manipulación (Fig. 8) de hormigón con forro interior inoxidable, y sometida a ventilación controlada. Esta celda está dotada de dos estaciones de trabajo, cada una con vidrios de plomo y dos telemanipuladores. La celda dispone de puente-grúa con telemanipulador pesado y pinza volteadora de bidones. Desde una de las estaciones de trabajo se controla el equipo de corte y eliminación de la envolvente metálica de los bidones y el equipo de ensayos mecánicos. Desde el segundo puesto se controla el sistema de trepanación de bidones y extracción de testigos en seco y el equipo de corte de probetas a partir de los testigos extraídos.
El laboratorio se completa con un sistema de caracterización radiológica de bultos (no destructiva), sistema de ensayos de aspersión y lixiviación de bidones (decorticados) y probetas, equipo de ensayos para el reglamento de transporte (compresión, caída), laboratorio químico y laboratorio de contaje.

\section{Planta de aguas residuales ${ }^{16}$}

Situada junto a la balsa cubierta de recogida de pluviales de la zona de edificios, está la planta de tratamiento de aguas residuales, para su tratamiento desde el punto de vista convencional.

\section{El acondicionamiento de los residuos}

Las operaciones realizadas en el edificio de acondicionamiento (Fig. 9) pueden agruparse en cinco líneas principales:

\subsection{Residuos de pequeños productores}

En la instalación se han incluido los sistemas y equipos necesarios para el acondicionamiento de los residuos de los pequeños productores. 
Entre los sistemas principales se incluye una caja de guantes para la clasificación, trituración, segregación y ensacado de residuos, así como un incinerador (Fig. 11) de $50 \mathrm{~kg} / \mathrm{h}$ para el tratamiento de los residuos biológicos y orgánicos.

El incinerador es del tipo de exceso de aire, con doble cámara de combustión. En la primera se alcanzan los $800^{\circ} \mathrm{C}$, y en la cámara de postcombustión se superan los $1.000^{\circ} \mathrm{C}$. A la salida de esta cámara hay un filtro de alta temperatura, de carburo de silicio. Posteriormente, los humos se enfrían mediante la dilución en aire frío hasta alcanzar los $140^{\circ}$ C. Los gases pasan entonces por los filtros de muy alta eficacia, descargándose, una vez filtrados, por la chimenea.

El incinerador puede ser alimentado con sólidos previamente triturados y ensacados en bolsas de cinco kg. Los líquidos se alimentan por bombeo desde un tanque de diario a través de un quemador que inyecta el líquido en la zona de postcombustión. Como combustible auxiliar para mantener las temperaturas se utiliza gasóleo. Se mantiene además una llama piloto mediante propano.

\subsection{Residuos compactables}

Se ha instalado una compactadora de bidones de $1.200 \mathrm{t}$ de fuerza, con la que se consiguen factores de reducción de volumen medios del orden de 3 (Fig. 10).

Los bidones con los residuos compactables se descargan, con un puente-grúa, en una nave específica para este tipo de bultos y se introducen en el equipo de compactación, pasando una sucesión de esclusas.

El equipo de compactación es una prensa hidráulica horizontal y la cámara de compactación está sometida a una fuerte depresión ( $40 \mathrm{~mm} \mathrm{ca}$.) mediante el sistema de ventilación controlada, que provoca simultáneamente un barrido para mantener bajas las concentraciones del recinto.

Los compactados o "pellets" se introducen mediante un equipo distribuidor que los coloca ordenadamente dentro de un contenedor de almacenamiento, antes de su envío a la línea de inyección de mortero.
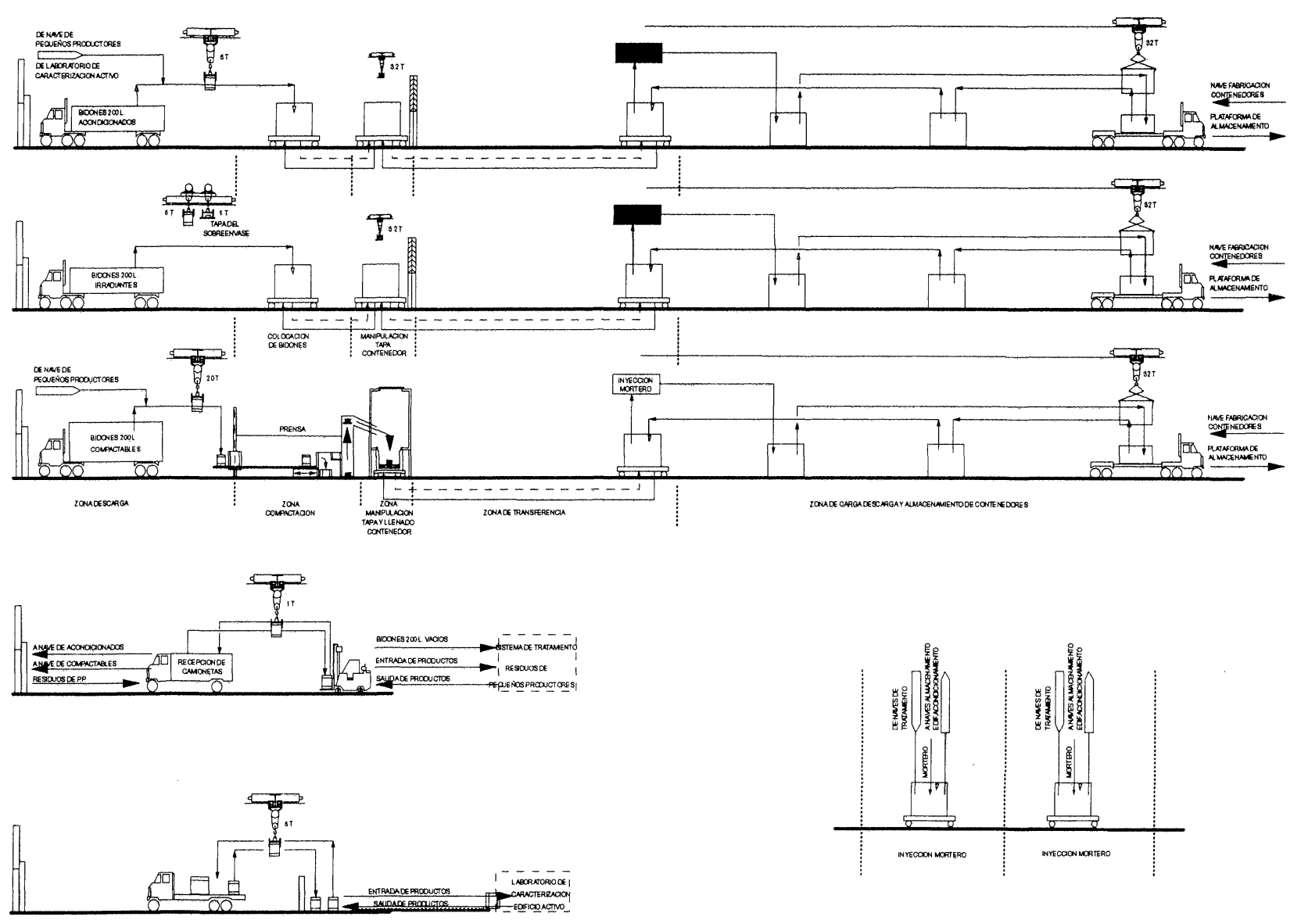

Fig. 10. Manutención edificio de acondicionamiento 


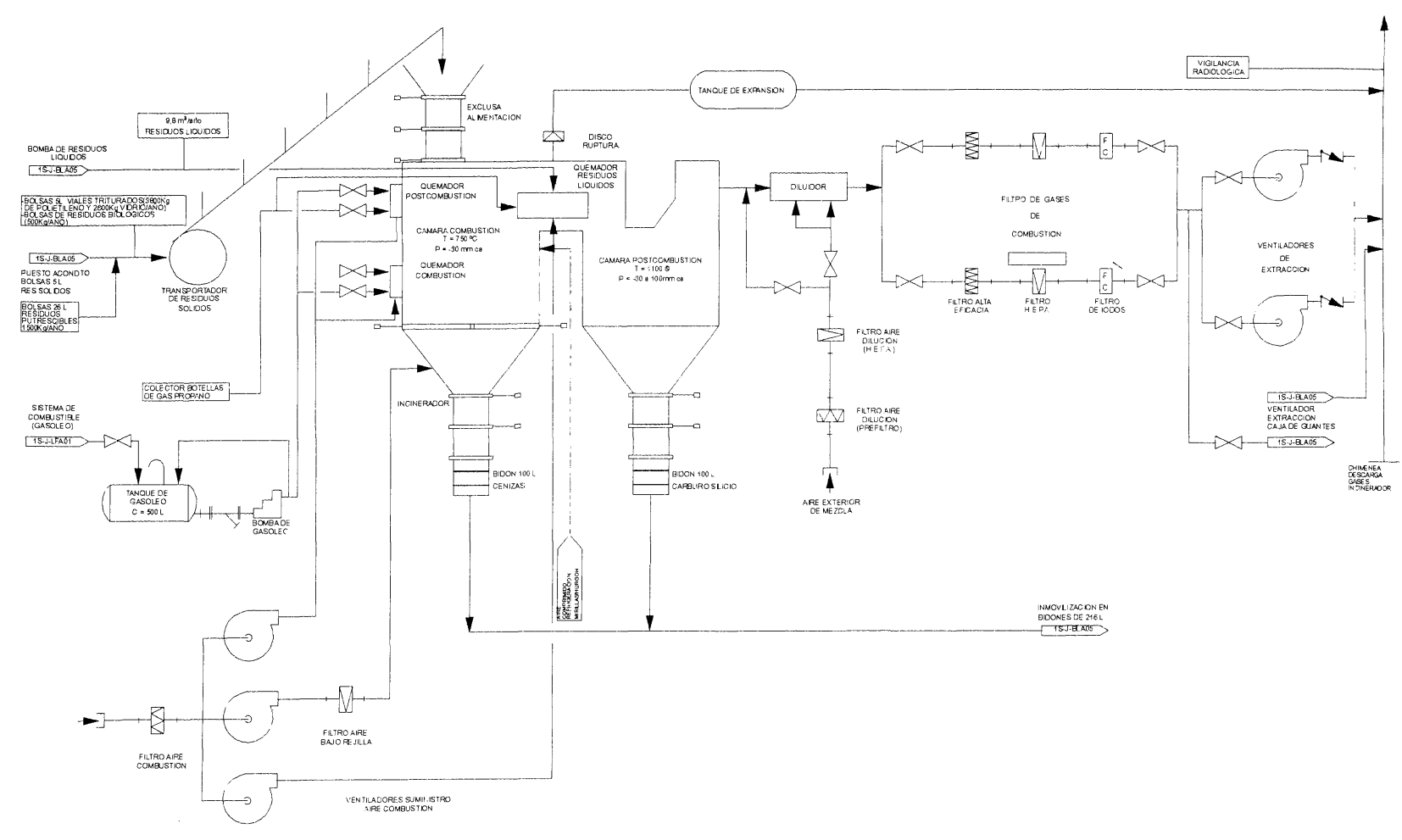

Fig. 11. Sistema de residuos P. P. (incineración)

Tanto la grúa de descarga, como la compactadora y el equipo de distribución son de funcionamiento semiautomático y se mandan desde la Sala de Control.

\subsection{Residuos inmovilizados}

Los residuos que llegan ya acondicionados en una matriz sólida, normalmente de cemento, se transfieren a los contenedores de almacenamiento con ayuda de un puente-grúa mandado remotamente. Hay dos naves similares para este tipo de residuos, diferenciándose entre sí en los equipos de elevación. Una, está dedicada a los bultos débilmente irradiantes, que llegan al centro sin blindaje adicional; y en otra se descargan los bultos que, por su tasa de dosis, se transportan en el interior de un blindaje adicional. Esta segunda nave presenta espesores mayores de blindaje y está dotada de elementos para la apertura del sobreenvase y el manejo de su tapa.

En ambos casos, una vez lleno el contenedor, éste se transfiere a la nave de manejo de contenedores mediante un carretón. Desde esta posición y, una vez colocada la tapa, el contenedor se transporta con el puente-grúa de la nave de contenedores, al puesto de inyección de mortero.

\subsection{Residuos generados en el Centro}

- Residuos líquidos.

Los residuos líquidos que pueden esperarse son soluciones acuosas de baja concentración y actividad y se recogen en unos depósitos situados en el sótano del edificio.

El sistema de tratamiento se ha seleccionado con dos objetivos: no aumentar el volumen de residuos a almacenar y cumplir el objetivo de vertido nulo de residuos líquidos.

Estos residuos líquidos, se incorporan previo análisis al mortero de inmovilización que llena los intersticios entre bidones, dentro del contenedor de almacenamiento.

De esta forma no se disminuye la capacidad de almacenamiento y, dado que la cantidad de agua requerida para la preparación de mortero es muy superior a la cantidad esperada de efluentes líquidos, se puede cumplir el objetivo de vertido nulo.

Los residuos líquidos que se obtienen en la compactación se decantan, separándose los aceites de los residuos acuosos. Éstos se envían a los depósitos de residuos líquidos. Los aceites se incineran. 
— Residuos sólidos.

Los residuos sólidos que se producen en la Instalación se tratan como compactables.

\subsection{Inyección de mortero}

Todos los contenedores, una vez colocada la tapa, se transfieren al puesto de inyección de mortero de inmovilización.

En el sistema de inyección se mezcla (Fig. 12) el mortero seco (mezcla de cemento y arena) con agua y aditivos. El mortero húmedo resultante se inyecta mediante bombas peristálticas a través de un inyector telescópico.

Excepto el manejo de los residuos de pequeños productores, que presentan en general tasas de dosis en contacto de fondo, todos los sistemas se operan con mando manual remoto desde la Sala de Control.

\section{Desarrollo del proyecto}

Una instalación como la de El Cabril debe reunir los requisitos de calidad y de demostración de la calidad inherentes a toda instalación nuclear, pero, además, presenta la característica de la singularidad, con múltiples procesos muy específicos que, aun probados industrialmente, han requerido un esfuerzo de desarrollo e ingeniería para su adaptación a los requisitos de esta instalación.

Ha sido una preocupación constante de ENRESA el mantener el mayor nivel de calidad en los materiales, así como una organización suficiente para garantizar la calidad de los diversos procesos de ingeniería, aprovisionamientos, construcción, montaje y pruebas.

Los principales hitos del proyecto se indican a continuación:

- A principios de 1986 se inician los trabajos de caracterización del emplazamiento, partiendo de los estudios realizados hasta entonces por el CIEMAT.

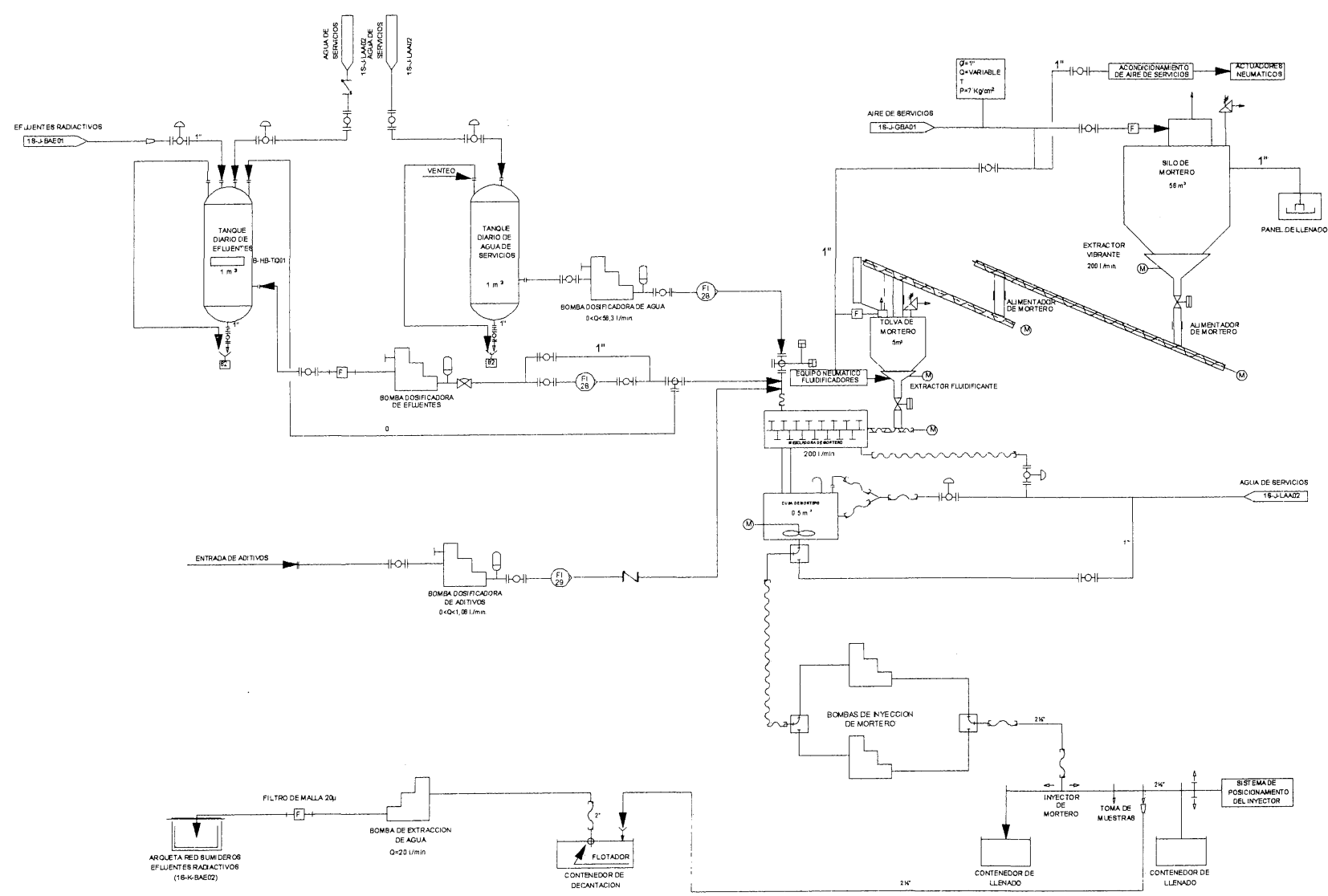

Fig. 12. Sistema de bloqueo de residuos 
- En la segunda mitad de 1986 se inician, en colaboración con la agencia francesa de gestión de residuos, ANDRA, los documentos preliminares de ingeniería.

- En mayo de 1987 se comienza el proyecto de Instalación, que es desarrollado por INITEC, con

- la asesoría de la ingeniería francesa TECHNICATOME.

- En mayo de 1988 se presenta la solicitud de autorización de construcción, de acuerdo con el Reglamento de Instalaciones Nucleares y Radiactivas, con la novedad de incluir un estudio de impacto ambiental, de acuerdo con el Decreto Legislativo sobre evaluación de impacto ambiental de 1987.

- En abril de 1989 se presenta una revisión general de la documentación preceptiva, para incluir el criterio de recuperabilidad de los residuos, después de diversas reuniones con el Consejo de Seguridad Nuclear y la Dirección General de la Energía.

- En mayo de 1989 se solicita, igualmente, la licencia municipal de obras al Ayuntamiento de Hornachuelos.

- En julio de 1989 el Consejo de Seguridad Nuclear emite el preceptivo informe favorable y en agosto la Dirección General del Medio Ambiente, de forma coordinada con el Consejo, emite la Declaración del Impacto Ambiental. Esta instalación ha sido el primer proyecto industrial sometido a dicha declaración.

- El Boletín Oficial del Estado del 2 de noviembre de 1989 publicaba la Orden del 31 de octubre de Autorización de Construcción. El 17 del mismo mes la Comisión Provincial de Urbanismo de Córdoba emitía su informe favorable a la licencia urbanística solicitada y, por fin, el Ayuntamiento de Hornachuelos aprobaba el 5 de diciembre la concesión de la licencia municipal, que era comunicada a ENRESA el 13 de diciembre.

- En enero de 1990 se iniciaban las excavaciones. La edificación, en octubre del mismo año.

- En abril de 1991 se presentó la solicitud de aprobación del Programa de Pruebas Prenucleares, y en mayo el Permiso de Explotación Provisional.

- En octubre de 1991 se energizó la instalación, iniciándose las pruebas, y en marzo de 1992 se realizó la primera de las pruebas oficiales del mencionado Programa de Pruebas Prenucleares. Este programa se dio por finalizado, con la excepción de lo referente al Laboratorio de Caracterización Activo, el 21 de julio de 1992.

- El 9 de agosto de 1992 se recibió la inspección final, previa al permiso, del Consejo de Seguridad Nuclear.

- El 20 de octubre de 1992 se emitió la Orden Ministerial de Permiso de Explotación Provisional, previo informe favorable del Consejo. Como parte de esta autorización estaba la de fabricación de los contenedores.

- A partir de noviembre de 1992 comenzó la operación propiamente dicha, en su fase de pruebas nucleares. En enero de 1993 se realizaron los ensayos del TPC y funcionales solicitados por el CSN para autorizar el uso de los contenedores de hormigón, comenzando el almacenamiento de éstos en las celdas a partir de abril de 1993. En marzo y abril de 1993 se realizaron las pruebas prenucleares del Laboratoio Activo de Verificación de la Calidad, cuya operación fue autorizada en julio de 1993.

- En junio de 1993 finalizó el programa de pruebas nucleares de la instalación entrando en operación normal.
1. Orden Ministerial de 31 de octubre de 1989 por la que se otorga a ENRESA la autorización de construcción del Proyecto de Ampliación de la instalación de almacenamiento de residuos radiactivos de sierra Albarrana. $B O E$ de 2-11-89.
2. Orden Ministerial de 9 de octubre de 1992 por la que se concede a ENRESA el Permiso de Explotación Provisional de la Ampliación de la Instalación de Residuos Radioactivos de sierra Albarrana. BOE de 21-10-92. 